that unlike most of the concentrated nickel Tutton salts $-\left(\alpha_{1}-\alpha_{11}\right)$ decreases with fall of temperature, bringing out prominently the temperature sensitiveness of the field coefficients and thus supporting the findings of Bose et al. ${ }^{3}$.

A. MoOKHERJI

R. B. LAL *

Physics Laboratories,

Burdwan University, India.

* At present post-doctoral research associate of the National Academy of Sciences, $R-R P-T$, Marshall Space Flight Center, Huntsville, Alabama.

${ }^{1}$ Joglekar, M. S., Z. F. Phys., 98, 411 (1938).

${ }^{2}$ Griffiths, J. H. E., and Owen, J., Proc. Roy. Soc., A, 213, 459 (1958)

sose, A., Mitra, S. C., and Datta, S. K., Proc. Roy. Soc., A, 248, 153 (1958).

- Krishnan, K. S., and Banerji, S., Phil. Trans. Roy. Soc., A, 234, 265 (1935).

'Krishnan, K. S., Mookherji, A., and Bose, A., Phil. Trans. Roy. Soc., A, 238, $125(1939)$.

Bose, A., Datta Roy, S. K., Ghose, P. K., and Mitra, S., Ind. J. Phys., 37, (1963).

'Mookherji, A., Ind. J. Phys., 19, 63 (1945).

\section{Light Scattering by the Relativistic (Non-linear)}

A SIMPLe non-linear model of the scattering particle is the relativistic oscillator (without damping), the approximate equation of motion of which ${ }^{1}$ is:

$$
\ddot{x}+\omega_{0}^{2} x-\varepsilon x \dot{x}^{2}=\left(F / m_{0}\right) \cos \omega_{1} t
$$

Here $\varepsilon=(3 / 2) \omega_{0}^{2} / c^{2}, \omega_{0}$ being the linear frequency and $m_{0}$ the rest mass. Assuming $F$ of the form $\varepsilon F_{0}$, a solution is $x=A \cos \omega_{1} t$, where:

$$
A=\frac{F_{0} / m_{0}}{\omega_{0}{ }^{2}-\omega_{1}{ }^{2}-\frac{\varepsilon \omega_{1}^{2}}{4} A^{2}}
$$

Setting $F_{0}=e E_{0}, e$ being electric charge and $E_{0}$ the field strength, the electric dipole moment $p=e x$, the average rate of emission $\mathrm{d} \bar{U} / \mathrm{d} t=\frac{\omega^{4} p^{2}}{3 c^{3}}$, and the intensity $\bar{I}=$ $(c / 8 \pi) E_{0}{ }^{2}$, we find for the ratio of incident to emitted intensity (for $N$ oscillators):

$$
\frac{\bar{I}}{\bar{I}_{0}} \simeq \frac{8 \pi N e^{4}}{3 m_{0}^{2} c^{4}} \frac{1}{\frac{\omega_{0}^{4}}{\omega_{1}^{4}}\left[1-\frac{3 \omega_{1}^{2} A^{2}}{8 c^{2}}\right]^{2}}
$$

For Thomson scattering, $\omega_{0} \ll \omega_{1}$ :

$$
\bar{I} / \bar{I}_{0} \simeq \frac{8 \pi N e^{4}}{3 m_{0}^{2} c^{4}}
$$

the cross-section remains constant as in the linear case.

For Rayleigh scattering, $\omega_{0} \gg \omega_{1}$ :

$$
\bar{I} / \bar{I}_{0} \simeq \frac{8 \pi N e^{4}}{3 m_{0}{ }^{2} c^{4} \omega_{0}{ }^{4}} \cdot \frac{\omega_{1}{ }^{4}}{\left[1-\frac{3 \omega_{1}{ }^{2} A^{2}}{8 c^{2}}\right]^{2}}
$$

The Rayleigh scattering cross-section decreases with the amplitude of the incident radiation of frequency $\omega_{1}$.

Using a ruby-laser beam, the Rayleigh scattering crosssection has been found ${ }^{2}$ to be smaller than the classical value by a factor of $10^{3}$. With the energy flux of $6 \times 10^{20}$ ergs $/ \mathrm{cm}^{2} / \mathrm{sec}$ as a typical value, the observed reduction in cross-section is of the same order of magnitude as predicted by the formula.

Note added in proof. Formulae similar to (2.2) may be derived also for the types of oscillator for which the nonlinear term is proportional to $x^{2}$ or to $x^{3}$. However, a numerical estimate depends on the value assumed for the factor of proportionality. Thus, the reduction in Rayleigh cross-section appears to express the amplitude-frequency relationship that characterizes any non-linear oscillator.

\section{Royal Holloway College, \\ University of London, \\ Englefield Green, \\ Surrey.}

E. H. Hutten

${ }^{3}$ Hutten, E. H., Nature, 205, 892 (1965).

${ }^{2}$ George. T. V., et. al.. Phus. Rev. Letters. 11.403 (1963).

\section{METALLURGY}

\section{A Metastable Intermediate Phase in the System Indium-Indium Antimony}

UsING a technique for the ultra-fast quenching of thin metal foils from the melt by solid conduction (splat cooling) ${ }^{1,2}$, we have recently shown ${ }^{3}$ that in many tin alloy systems a metastable phase of the ' $\mathrm{HgSn}_{6-10}$ ' type ${ }^{4}$ $(\gamma$-phase, simple hexagonal, 1 atom/cell, $a=3.212 \AA$; $c / a=0.9312)$ can be obtained. This phase exists in equilibrium in the systems Sn-In, Sn-Cd and Sn-Hg, and could be retained in non-equilibrium at $-190^{\circ} \mathrm{C}$ in the systems $\mathrm{Sn}+\mathrm{Pb}, \mathrm{Ga}, \mathrm{Zn}, \mathrm{Cu}, \mathrm{Ag}, \mathrm{Au}, \mathrm{Pd}, \mathrm{Ca}$ and $\mathrm{Mg}$, at solute additions of 5-20 atom per cent, depending on the system. All these elements, in leading to the formation of $\gamma$, acted to reduce the valence electron concentration of tin to less than $4 \cdot 0$.

InSb resembles $\mathrm{Sn}$ in some crystal-chemical respects: identical tetrahedral bonding in $\alpha$-Sn $(A 4)$ and InSb (B3); existence of a metallic high-pressure modification of InSb with the $\beta$-Sn structure ${ }^{5}$; and identical position in the periodic table. This made it likely that a metallic phase of the $\gamma$-type could also be retained from a melt of InSb with a suitable addition element, if the formation of equilibrium InSb could be partly suppressed.

The eutectic system In-InSb was selected. Alloys of In $+10,15,20,30$ and 40 atomic per cent $\mathrm{Sb}$ were rapidly quenched on to $\mathrm{Cu}$ or $\mathrm{Ag}$ substrates and investigated by $\mathrm{X}$-ray diffraction at $-190^{\circ} \mathrm{C}$ and $20^{\circ} \mathrm{C}$, as described in detail in ref. 3. Especially in the alloy In ${ }_{.70} \mathrm{Sb}_{.30}$, but in lesser degree also in $\mathrm{In}_{.80} \mathrm{Sb}_{.20}$ and $\mathrm{In}_{{ }_{60}} \mathrm{Sb}_{.40}$, twelve additional diffraction peaks were found. These lines could be indexed with a hexagonal unit cell of the $\gamma$-type $(a=3.205 \pm 0.003 \AA ; c=2.981 \pm 0.006 \AA ; c / a=$ $0.930+0.003$ at $-190^{\circ} \mathrm{C}$ ) and correspond closely in position and intensity to the powder pattern given by Screaton and Ferguson ${ }^{6}$ for the equilibrium $\gamma$-phase in In-Sn. From the relative intensities of In, $\gamma$ and InSb lines, it was estimated that with the present cooling rates $\left(10^{6}-10^{7}{ }^{\circ} \mathrm{C} / \mathrm{sec}\right.$ ) (ref. 2) about 50 per cent of the alloy $\mathrm{In}_{.70} \mathrm{Sb}_{.30}$ consisted of $\gamma$ and that the composition of $\gamma$ is $\mathrm{In}_{1-x} \mathrm{Sb}_{x}$ with $0.35<x<0.45$. The lines observed are the only lines expected and present in the investigated $2 \theta$ range from $20^{\circ}$ to $100^{\circ}$ (copper $K \alpha$ ); higher angle lines are not sharp enough due to transformation and texture effects. In general, while structure determinations based on few lines are problematic, in the case of metastable alloys or high-pressure phases no other evidence is available; for the In-InSb system, the analogy of the observed diffraction lines to those of the In-Sn $\gamma$-phase makes the reported result certain. At room temperature, the equilibrium structure In + InSb is established. Using the $c / a$-ratio observed for $\gamma$ of 0.930 and the table for valence electron concentration versus $c / a$ values in ref. $3, a$ valence electron concentration of $3.80 \mathrm{e} / \mathrm{a}$ is calculated for $\gamma(\mathrm{In}-[\mathrm{nSb})$. This would correspond to a concentration of $\operatorname{In}_{.60} \mathrm{Sb}_{.40}$ for $\gamma$, and would thus place $\gamma(\mathrm{In}-\mathrm{InSb})$ at approximately the same valence electron concentration as the corresponding equilibrium phase $\gamma(I n-S n)$. The observation that a maximum amount of $\gamma$ is obtained at $\mathrm{In}_{{ }_{70}} \mathrm{SB}_{30}$ rather than at $\mathrm{In}_{60} \mathrm{Sb}_{._{40}}$ can perhaps be explained on the assumption that an excess of In is necessary to prevent the formation of tetrahedrally bonded InSb nuclei on solidification, which would favour the equilibrium phases.

The formation of a second orthorhombic high-pressure phase of InSb ${ }^{5}$, in addition to the $\beta-S n$ type modification, with a unit cell closely related to the new $\gamma$-phase (distortion of the orthohexagonal basal plane of $\gamma$ with $b / a=\sqrt{ } 3$ into an orthogonal basal plane with $b / a \neq \sqrt{ } 3$ ) permits the speculation that in this high-pressure phase, just as in the new $\gamma$-phase, the effective valence electron concentration may be less than 4 .

There are also indications of the existence of a further metastable phase at the composition $\mathrm{In}_{.25} \mathrm{Sb}_{.75}$ (valence 